\title{
Patient-Refined Messaging for a Mailed Colorectal Cancer Screening Program: Findings from the PROMPT Study
}

\author{
Gloria D. Coronado, PhD, Jamie H. Thompson, MPH, Amanda F. Petrik, MS, \\ Denis B. Nyongesa, MS, Michael C. Leo, PhD, Melissa Castillo, MSW, \\ Brittany Younger, MSW, Anne Escaron, PhD, and Alex Chen, MD
}

Background: Improving uptake of colorectal cancer screening has the potential of saving thousands of lives. We compared the effectiveness of automated and live prompts and reminders as part of a mailed fecal immunochemical test (FIT) outreach program.

Design and Methods: Participants were 1767 adults aged 50 to 75 eyars who were not up-to-date with colorectal cancer screening recommendations at a participating community health center clinic. In addition to a mailed FIT kit, participants were randomized to receive (1) a text message prompt and 2 automated phone call reminders (automated condition); (2) up to 3 live call reminders (live condition); or (3) a text message prompt, 2 automated call reminders, and up to 3 live reminders (combined automated plus live condition). We assessed FIT completion rates in each group 6 months following randomization.

Key Results: Nearly one-third of participants completed an FIT within 6 months. Compared with adults allocated to the automated condition, FIT completion rates were higher in adults allocated to the live condition (32.3\% vs $26.0 \%$; adjusted difference, 6.3 percentage points; $95 \%$ CI, $1.1-11.4$ ) and in adults allocated to the combined automated plus live condition $(35.7 \%$ vs $26.0 \%$; adjusted difference, 9.7 percentage points; $95 \%$ CI, $4.4-14.9)$. The number of kits needed to mail to achieve a completed FIT ranged from 2.8 in the combined automated plus live condition to 3.8 in the automated condition.

Conclusions: Among unscreened individuals in this population, live phone call reminders either alone or in combination with automated prompts and reminders outperformed automated approaches alone. (J Am Board Fam Med 2019;32:318-328.)

Keywords: Colon Cancer, Colorectal Cancer, Community Health Centers, Early Detection of Cancer, Hispanic Americans, Occult Blood, Text Messaging

Screening for colorectal cancer (CRC) can substantially reduce CRC incidence and mortality. ${ }^{1,2}$ Sixty percent of the 50,000 annual deaths from CRC could be averted with routine screening. ${ }^{3}$ Unfortunately, screening rates are suboptimal in the gen-

This article was externally peer reviewed.

Submitted 17 September 2018; revised 31 January 2019; accepted 1 February 2019.

From Center for Health Research, Kaiser Permanente Northwest, Portland, OR (GDC, JHT, AFP, DBN, MCL); AltaMed Health Services, Los Angeles, CA (MC, BY, AE, AC).

Funding: Research reported in this publication was supported by the National Institutes of Health National Institute of Minority Health and Health Disparities through award U01MD010665.

Conflict of interest: From November 2014 to August 2015, Dr. Coronado. served as a coinvestigator on an industryfunded study to evaluate patient adherence to an experimental blood test for colorectal cancer. The study was funded by eral population and are especially low in community health centers. Uniform Data Systems data from 2016 show that only $40 \%$ of eligible adults in

EpiGenomics. From September 2017 to June 2019, Dr. Coronado has served as the principal investigator on an industry-funded study to compare the clinical performance of an experimental FIT to a Food and Drug Administrationapproved FIT. This study is funded by Quidel Corporation. All other authors declare no conflicts of interest.

Corresponding author: Gloria D. Coronado, PhD, Center for Health Research, Kaiser Permanente Northwest, 3800 North Interstate Avenue, Portland, OR, 97227 (E-mail: Gloria.D.Coronado@kpchr.org).

Trial Registration: National Clinical Trial (NCT) Identifier NCT03167125.

See Related Article on Page 307. 
community health centers were up-to-date with CRC screening recommendations. ${ }^{4}$ Mailed fecal immunochemical test (FIT) outreach programs have been shown to improve CRC screening rates in community health centers, ${ }^{5-11}$ with absolute improvements ranging from $22 \%$ to $45 \% .{ }^{5-11}$ Typically, these programs send patients prompts (before the kit is received) and/or reminders (after the kit is received) in the form of letters, telephone calls, or text messages in addition to sending information along with the mailed FIT kit., ${ }^{5,10-13}$ These prompts and reminders are assumed to motivate patients to complete the FIT, thus increasing response rates and program efficacy. However, despite the widespread use of prompts and reminders for mailed FIT outreach programs, data are sparse on how these communications influence FIT completion rates.

Data from our previous work compared reminder letters to automated and live phone calls and text messages delivered after community health center patients had received an FIT kit in the mail. We found a $50 \%$ higher FIT completion rate among patients who received a live phone call reminder than those that received a reminder letter (odds ratio [OR], 1.51, 95\% CI [1.03 - 2.21]) but no significant benefit for automated reminder calls or text messages. ${ }^{14} \mathrm{We}$ also found that the effectiveness of a given reminder varied by patients' language preference: while the live phone call condition was the most effective for patients who preferred English, patients who preferred Spanish were most likely to return their FIT when presented with the combination of automated plus live phone calls. Although this work suggests that patients respond better to phone calls than reminder letters after being mailed a FIT kit, the study did not send patients any prompts before the FIT kit was received. Furthermore, the content of reminder messages was chosen by researchers without input from patient populations.

The present study compares the effectiveness of combinations of prompts and reminders to a mailed FIT outreach program administered in 2 clinics operated by a large, Los Angeles-based community health center. Prompt and reminder content were developed and refined by patient stakeholders using a validated engagement process - boot camp translation (see companion article). ${ }^{15} \mathrm{We}$ also assessed how the effects of the prompts and reminders varied across patient demographic characteristics and health care use patterns. The results of this pilot study will inform future efforts to optimize FIT return rates for mailed outreach programs.

\section{Methods \\ Study Setting}

The pilot study was conducted at 2 clinics within a large Los Angeles- and Orange County-based community health center that operates 27 medical clinics and served more than 280,000 patients in 2017, including 22,705 between the ages of 50 to 75 . The clinics were chosen in partnership with clinic staff, with the goal of selecting a representative clinic in each county and having enough patients to enroll in the pilot study. Over $97 \%$ of health center patients aged 50 to 75 preferred speaking English or Spanish. The health center screened $67 \%$ of all eligible patients for CRC in 2017. The proportion uninsured was $18.0 \%$. The health center has near-complete capture of FIT events in the electronic health record (EHR) as FIT results are transferred from the reference laboratory through a direct electronic interface. The health center engages in various efforts to promote the use of FIT among clinic patients. These include routine FIT distribution during office visits, videos in waiting rooms, and a mailed FIT program that involves live telephone call reminders delivered by clinic medical assistants.

The findings of this pilot study will inform the design of the main trial that will test the implementation and effectiveness of an optimized, mailed FIT and reminder intervention across 15 health center clinics. All procedures and intervention materials were reviewed and approved by the Institutional Review Board of Kaiser Permanente Northwest, with ceding agreements from the health center and Oregon Health \& Science University. The study obtained a waiver of informed consent, given the minimal risk posed to patients.

\section{Development and Content of Prompts and Reminders}

We used boot camp translation, ${ }^{16}$ a well-validated patient engagement approach, to develop the content and select the format of prompts and reminders for this study. The study's boot camp translation methods have been reported previously (see companion article Thompson et al JABFM 180026). ${ }^{15,17}$ Briefly, we recruited 25 patients and 4 
clinic staff to participate 2 parallel boot camp translation programs, 1 in English and 1 in Spanish. Each program included a 6-hour in-person session that consisted of an expert presentation and interactive large- and small-group activities aimed at developing initial drafts of outreach materials, and 330 - to 45-minute group conference calls facilitated by a member of the project team. The goal of the conference calls was to solicit patient feedback on draft program outreach materials designed based on the in-person sessions. The materials included a text message, an introductory letter, a 1-page pictographic educational fact sheet, and automated and live reminder phone call scripts. The sessions and conference calls were held over 3 months (May 12-July 12, 2017).

All materials were developed in English and Spanish. The FIT kit packet contained an introductory letter, a 1-page pictographic educational sheet, a FIT kit, written instructions (in English and Spanish) on how to complete the kit, and a postage-paid envelope for returning the kit to the health center's contracted laboratory. The introductory letter explained the importance of CRC screening, stated that testing was free, and emphasized the importance of completing the test. The 1-page pictographic educational sheet provided information about the importance of CRC and the need for screening. The text message prompt informed recipients that they would be mailed a FIT and provided the clinic number to call if they had questions. Scripts for the live call included sample dialog and responses to commonly asked questions. All materials were reviewed by bilingual staff. The FIT used was InSure (Clinical Genomics; Bridgewater, $\mathrm{NJ}$ ), a 2 -specimen FIT that requires no dietary restrictions.

\section{Study Procedures}

Centralized clinic outreach staff used a list of EHR codes (eg, diagnoses codes, procedure codes, and laboratory codes) from previous studies ${ }^{18}$ to identify adults who were overdue for CRC screening and had attended at least 2 clinic visits within the past 24 months. ${ }^{19}$ A total of 3201 adults in the 2 participating clinics met the criteria. Using this list, clinic staff removed 1218 individuals whose insurance status could not be validated and their patient ("enrollment") status could not be confirmed ( $\mathrm{n}=$ 990), had an invalid address $(\mathrm{n}=107)$, or were otherwise ineligible, for example, had a total colec- tomy or CRC $(\mathrm{n}=147$; note that reasons were not mutually exclusive). Of the remaining 1983 patients, 216 were mailed a FIT but not sent any prompts or reminders because they were on the "do not contact" list (patients can opt to be added to this list during any telephone or in-person conversation with clinic staff). This left 1767 adults (678 from clinic A and 1089 from clinic B) who were randomized into the 3 study conditions ( $\mathrm{n}=589$ per arm). Randomization was stratified by clinic.

\section{Intervention Conditions}

This study included 3 conditions, providing (1) automated, (2) live, and (3) combined automated plus live prompts and reminders as part of a mailed FIT outreach program. Patients in all 3 conditions were mailed an FIT kit with an introductory letter and 1-page educational sheet on 7/5/2017.

1. Patients in the automated condition were sent a text message prompt on $7 / 8 / 2017$ (before the patient was expected to receive the kit), and 2 automated phone call reminders were delivered on 9/13/17 and 9/15/17. Automated phone calls and text messages were delivered in English or Spanish (depending the patients' language indicated in the EHR), between the hours of 10:00 AM and 9:00 PM Monday through Friday. The automated call reminded patients to complete the colon health test they were sent and invited patients to press " 0 " to be transferred immediately to speak to a staff member at the health center's Patient Contact Center. Texts and automated calls were sent by a contracted vendor (Stericycle Communication Solutions); the outgoing phone number was a toll-free 888 number.

2. Patients in the live condition received up to 3 live phone call reminder attempts delivered by centralized clinic outreach staff between 9/19/17 and $10 / 20 / 17$ by using a patient list that had been refreshed weekly from $9 / 18 / 17$ through $10 / 16 / 17$ to remove patients who had completed their FIT and needed no further reminders. Outreach staff were bilingual in English and Spanish, and interpreter services were available for patients who spoke other languages. Live calls were made between the hours of 8:00 AM and 5:00 рм Monday through Friday to the contact phone number in the patients' EHR. Patients who were left a voicemail received subsequent reminder attempts (up to 3); patients who were reached in-person or whose contact information was incorrect received no further reminder 
attempts. The outgoing phone number was a local clinic number.

3. Patients in the combined condition received the text message prompt on 7/8/2017, 2 automated phone calls, and up to 3 live phone call reminders, delivered on the same schedule as the automated condition and live condition (described above).

The intervention was not delivered as intended for a small subset of patients as 42 (of 589) patients allocated to the automated condition were delivered automated plus live call reminders by clinic staff. However, in accordance with intention-totreat principles, these participants remained in the group they were originally assigned.

\section{Statistical Analysis}

We describe the demographic characteristics of all randomized patients across each intervention condition and the percentage of FIT kits completed within 6 months of randomization. To identify how completion rates varied by patient characteristics, we used Wald tests and multivariable logistic regression to assess bivariate and multivariate associations between FIT completion and patient sociodemographic characteristics (age, sex, ethnicity, language, insurance status, and clinic) and health care use information (number of clinic visits in the past year, number of no-shows in the past year, and prior fecal testing).

Our primary intention-to-treat analysis compared FIT completion rates across treatment conditions by using multiple logistic regression, with FIT completion as the dependent variable, treatment condition as the independent variable (dummy coded with the automated arm as the reference group), and site (clinic A or clinic B) as a covariate. We calculated reach as the proportion of patients who received a given prompt or reminder that they were randomized to receive (eg, text message was successfully sent, automated phone call was delivered, a live phone call conversation took place, or a message was left). Using these data, we performed 2 types of per-protocol analysis. The first performed the same comparisons as the primary outcome analysis and was limited to patients who received all steps in the intervention as intended (eg, received text message, automated phone calls, and live phone call). A second perprotocol analysis assessed return rates based on whether patients received a given prompt or reminder. For this second analysis of live calls, we excluded patients who completed an FIT and did not need a live call reminder. We also assessed the number of kits needed to mail to achieve a completed FIT for each arm.

Finally, we examined whether preferred language (English or Spanish, or other), presence of a clinic visit in past year (no visits or 1 or more visits), and history of an FIT (never had a FIT or had at least 1 FIT in the past) modified the treatment effect by performing additional multiple logistic regressions including the product of each condition vector and the moderator variable (separate regressions for language, presence of a clinic visit, and history of an FIT). To assess the impact on our findings of the 42 patients assigned the automated outreach who were mistakenly delivered automated plus live outreach, we conducted a sensitivity analysis excluding those patients.

\section{Results}

A total of 1767 patients were randomized to the 3 intervention conditions ( $\mathrm{n}=589$ per arm; Figure 1). Randomized patients had a mean age of 59 years (SD, 5.7) and 57\% were female (Table 1). Latinos represented $83 \%$ of the sample. Most patients preferred speaking Spanish (59\%) and were insured by Medicaid/Medicare (57\%); on average, they had visited the clinic 3 times in the past year (SD, 3.1). Nearly two-thirds no-showed for a clinic visit in the past year and one-half had EHR evidence of having ever completed a prior FIT. Patients in clinic A and clinic B were similar in demographic and health care use characteristics.

Overall, 553 patients completed their FIT, for a return rate of $31.3 \%$. Crude FIT completion rates were $26.0 \%$ in the automated condition, $32.3 \%$ in the live condition, and $35.7 \%$ in the automated plus live condition (Table 2). Controlling for clinic, adults allocated to the live condition had a significantly higher FIT completion rate than those in the automated condition $(32.3 \%$ vs $26.0 \%$; adjusted difference, 6.3 percentage points; 95\% CI, 1.111.4), as did adults allocated to the combined automated plus live condition $(35.7 \%$ vs $26.0 \%$; adjusted difference, 9.7 percentage points; $95 \%$ CI, 4.4-14.). Adjusted differences increased in per-protocol analysis for both comparisons: live versus automated (36.3\% vs $29.3 \%$; adjusted difference, 6.9 percentage points; 95\% CI, 0.6-13.4), and combined automated plus live versus automated (41.4\% 
Figure 1. CONSORT diagram. FIT, fecal immunochemical test.

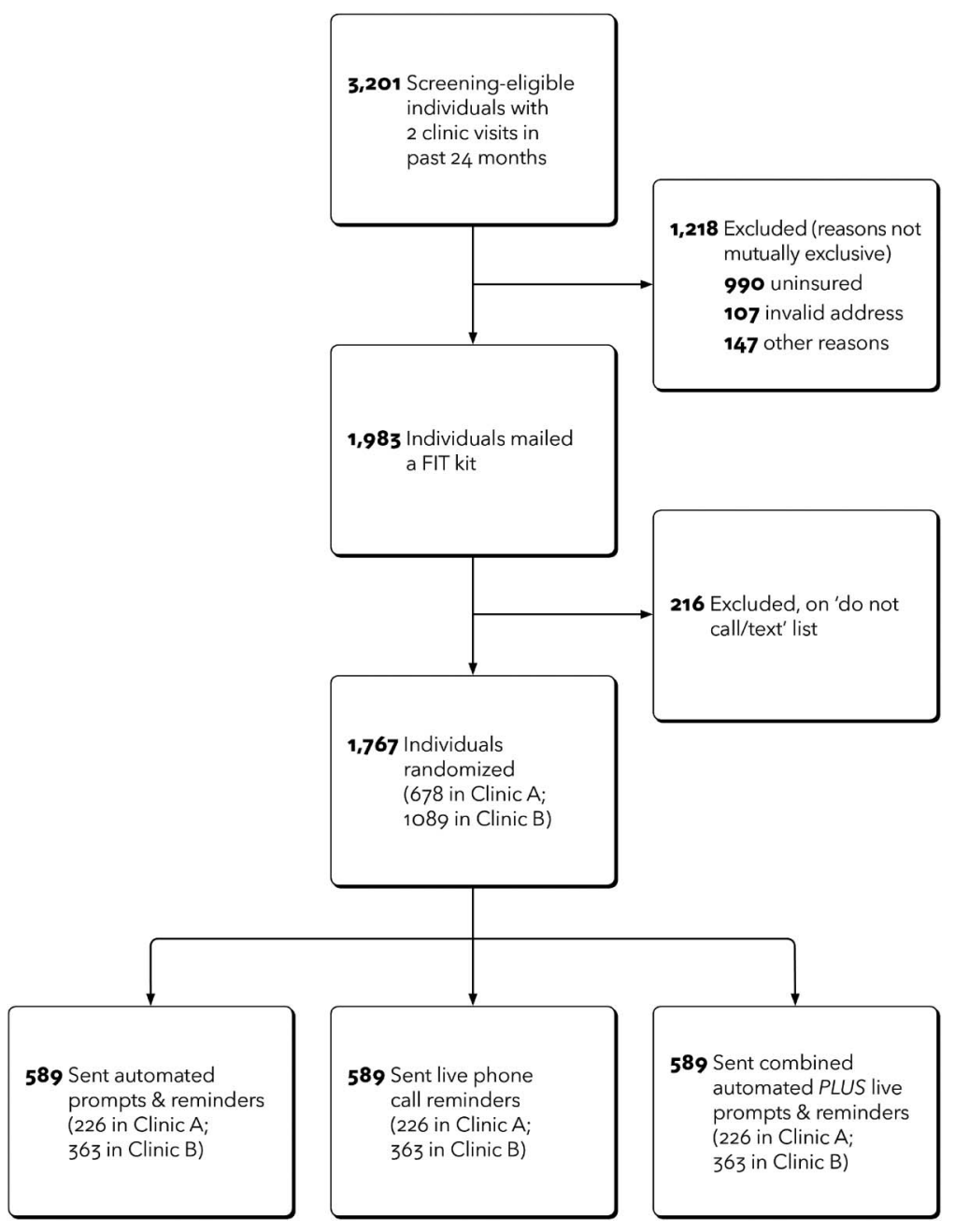

vs $29.3 \%$; adjusted difference, 12.1 percentage points; 95\% CI, 4.9-19.2). The number of kits needed to mail to achieve a completed FIT ranged from 2.8 in the combined automated plus live condition to 3.8 in the automated condition.

Reach was highest for text messages $(80 \%$ in the automated condition; $81 \%$ in the automated plus live condition) and automated calls (74\% in the automated condition; $77 \%$ in the automated plus live condition), and lower for live calls (51\% in the live condition; $55 \%$ in the automated plus live condition) (Table 3). Among the 888 adults reached via an automated phone call $(\mathrm{n}=436$ in the automated condition and $n=452$ in the automated plus live condition), $16.9 \%$ were reached in-person; among the 624 reached via a live call $(n=324$ in the live arm and $n=300$ in the automated plus live arm), $67.8 \%$ were reached in-person. Notably, our assessment of reach for automated calls and text mes- sages relied on vendor tracking information, which could not confirm that an automated phone message was delivered to the intended recipients or whether a given phone enabled text-message functions. In analyses of the intervention components, FIT completion proportions were similar among patients who did and did not receive the text message prompt $(26 \%$ vs $25 \%, P=.70$ in the automated condition; $35 \%$ vs $37 \%, P=.69$ in the automated plus live condition) but were significantly higher for patients who received the automated phone call reminder versus not $(28 \%$ vs $17 \%, P=.01$ in the automated condition; $38 \%$ vs $24 \%, P<.01$ for the automated plus live condition) or live phone call reminder versus not $(22 \%$ vs $13 \%, P=.05$, for the live condition; $22 \%$ vs $9 \% . P<.01$ for the automated plus live condition). FIT completion rates were higher for phone calls that were answered in-person versus by machine 
Table 1. Baseline Electronic Health Record-Derived Demographic and Health Care Use Characteristics of Selected Adults Due for Colorectal Cancer Ccreening $(n=1767)$ Who Attended at Least 2 Clinic Visits in the Past 24 Months, Intention-to-Treat Analysis Sample

\begin{tabular}{|c|c|c|c|}
\hline \multirow[b]{2}{*}{ Characteristic } & \multicolumn{3}{|c|}{ Randomized Patients in Clinic A and Clinic B } \\
\hline & $\begin{array}{c}\text { Clinic A } \\
(\mathrm{n}=678) \\
\mathrm{N}(\%)\end{array}$ & $\begin{array}{c}\text { Clinic B } \\
(\mathrm{n}=1089) \\
\mathrm{N}(\%)\end{array}$ & $\begin{array}{c}\text { Total } \\
(\mathrm{n}=1767) \\
\mathrm{N}(\%)\end{array}$ \\
\hline \multicolumn{4}{|l|}{ Age $^{*}$} \\
\hline Mean (SD) & $58.6(5.9)$ & $58.6(5.5)$ & $58.6(5.7)$ \\
\hline 50 to 64 & $558(82.3)$ & $939(86.2)$ & $1497(84.7)$ \\
\hline 65 to 74 & $113(16.7)$ & $145(13.3)$ & $258(14.6)$ \\
\hline \multicolumn{4}{|l|}{$\operatorname{Sex}^{\dagger}$} \\
\hline Female & $385(56.8)$ & $620(56.9)$ & $1005(56.9)$ \\
\hline Male & $292(43.1)$ & $469(43.1)$ & $761(43.1)$ \\
\hline \multicolumn{4}{|l|}{ Ethnicity $^{\ddagger}$} \\
\hline Non-Hispanic/Latino & $163(24.0)$ & $111(10.2)$ & $274(15.5)$ \\
\hline Hispanic/Latino & $506(74.6)$ & $966(88.7)$ & $1472(83.3)$ \\
\hline \multicolumn{4}{|l|}{ Language $^{\S}$} \\
\hline English & $223(32.9)$ & $458(42.1)$ & $681(38.5)$ \\
\hline Spanish & $438(64.6)$ & $611(56.1)$ & $1049(59.4)$ \\
\hline Other & $16(2.4)$ & $18(1.7)$ & $34(1.9)$ \\
\hline \multicolumn{4}{|l|}{ Insurance Status } \\
\hline Medicaid/Medicare & $336(49.6)$ & $677(62.2)$ & $1013(57.3)$ \\
\hline Uninsured & $214(31.6)$ & $215(19.7)$ & $429(24.3)$ \\
\hline Commercial & $128(18.9)$ & $197(18.1)$ & $325(18.4)$ \\
\hline \multicolumn{4}{|l|}{ Clinic visits in past year } \\
\hline Mean (SD) & $3.0(2.8)$ & $3.0(3.3)$ & $3.0(3.1)$ \\
\hline No visits & $133(19.6)$ & $221(20.3)$ & $354(20.0)$ \\
\hline At least 1 visit & $545(80.4)$ & $868(79.7)$ & $1413(80.0)$ \\
\hline \multicolumn{4}{|l|}{ No show in past year } \\
\hline No & $269(39.7)$ & $330(30.3)$ & $599(33.9)$ \\
\hline Yes & $409(60.3)$ & $759(69.7)$ & $1168(66.1)$ \\
\hline \multicolumn{4}{|l|}{ Prior fecal testing } \\
\hline Never & $356(52.5)$ & $518(47.6)$ & $874(49.5)$ \\
\hline Ever & $322(47.5)$ & $571(52.4)$ & $893(50.5)$ \\
\hline \multicolumn{4}{|l|}{ Randomization arm } \\
\hline Automated & $226(33.3)$ & $363(33.3)$ & $589(33.3)$ \\
\hline Live & $226(33.3)$ & $363(33.3)$ & $589(33.3)$ \\
\hline Automated plus live & $226(33.3)$ & $363(33.3)$ & $589(33.3)$ \\
\hline
\end{tabular}

SD, standard deviation.

*12 values were missing (7 in clinic A; 5 in clinic B).

${ }^{\dagger} 1$ value was missing in clinic A.

${ }^{\ddagger} 21$ values were "other" or missing (9 in clinic A; 12 in clinic B).

$\$_{3}$ values were missing (1 in clinic A; 2 in clinic B).

( $47 \%$ vs $30 \%$ for automated calls; $29 \%$ vs $10 \%$ for live calls; data not shown). Reach was similar across clinics (text message: clinic A, $81 \%$; clinic B, $81 \%$; automated calls: clinic A, 75\%; clinic B, 76\%; live calls: clinic A, 52\%; clinic B, 54\%).

In bivariate comparisons, patient demographic and health care use characteristics associated with FIT completion were preferred language
(Spanish 35.2\% vs English 25.4\%; OR, 1.59, $95 \%$ CI $[1.29,1.97])$, clinic visits in the past year (at least 1 visit, $33.8 \%$ vs no visits, $21.2 \%$; OR, $1.90[1.44,2.51]$ ), and prior FIT testing (Yes, $45.1 \%$ vs No, $17.2 \%$; OR, 3.97, 95\% CI [3.19, 4.95]; Table 4). These variables remained significant in multivariable-adjusted models. No significant interactions were observed between 
treatment conditions and language, number of clinic visits, or prior FIT testing.

When we examined FIT completion rates in the automated condition with and without the $42 \mathrm{pa}-$ tients who were mistakenly delivered a live phone call reminder, we found a $26 \%$ completion rate with the patients included and a $27 \%$ completion rate without, suggesting that this error had negligible impact. Of the 553 adults who completed a FIT, $38(6.9 \%)$ had a positive test result.

\section{Discussion}

Our mailed FIT outreach intervention was designed with input from patients and clinicians, using boot camp translation (see companion article, Thompson et al JABFM 18-0026). ${ }^{17} \mathrm{We}$ found that delivering live telephone reminders after sending a mailed FIT, alone or in combination with text message prompts and automated telephone call reminders, can increase response rates by 6 to 9 percentage points compared with text message prompts and automated calls without a live phone component in a large, urban, primarily Latinoserving community health center. Overall, FIT completion rates were higher in patients whose preferred language was Spanish (vs English), who had 1 or more clinic visits in the past year (vs none), and who had completed a prior FIT (vs never completed an FIT). Our findings support the use of multiple touchpoints, including live phone reminders, to optimize FIT kit return rates in mailed outreach programs, and may inform efforts to tailor outreach approaches to population subgroups.

Consistent with our previous research and that of others, ${ }^{7,14}$ we observed that patients who preferred speaking Spanish had a higher FIT completion rate than patients who preferred speaking English. This may reflect Latino patients' greater willingness to follow provider recommendations, a tendency noted in previous research. ${ }^{20,21}$ However, these results stand in contrast to those of Baker and colleagues, ${ }^{5}$ who reported no differences in the effectiveness of the direct-mail and reminder intervention based on race/ethnicity or preferred language. Nevertheless, Bakers' study of 450 adults included relatively few English speakers (72;16\%) and non-Latino whites (54;12\%), limiting the power to make such comparisons. We also observed that patients who had 1 or more clinic visit in the past year had a higher FIT completion rate 
Table 3. Intervention Components Received and Their Association with Fecal Immunochemical Test Completion

\begin{tabular}{|c|c|c|c|}
\hline Randomization Condition & Reach, N (\%) & FIT Completion, \% & $P$ value* \\
\hline \multicolumn{4}{|l|}{ Condition 1: automated $^{\dagger}$} \\
\hline Text message prompt ${ }^{\S}$ & & & .70 \\
\hline Completed & $471(80.0)$ & 26.3 & \\
\hline Not completed & $118(20.0)$ & 24.6 & \\
\hline Automated phone call reminders & & & $<.01$ \\
\hline Answered in-person & $78(13.2)$ & 39.7 & \\
\hline Answered by machine & $358(60.8)$ & 25.7 & \\
\hline Not completed & $128(21.7)$ & 17.2 & \\
\hline No call needed & $25(-)$ & - & \\
\hline \multicolumn{4}{|l|}{ Condition 2: live phone call } \\
\hline Live phone call reminders & & & $<.01$ \\
\hline Answered in-person & $208(35.3)$ & 29.3 & \\
\hline Answered by machine & $116(19.7)$ & 7.8 & \\
\hline Not completed & $101(17.1)$ & 12.9 & \\
\hline No call needed & $164(-)$ & - & \\
\hline \multicolumn{4}{|c|}{ Condition 3: automated plus live phone call } \\
\hline Text message prompt ${ }^{\S}$ & & & .69 \\
\hline Completed & $479(81.3)$ & 35.3 & \\
\hline Not completed & $110(18.7)$ & 37.3 & \\
\hline Automated phone call reminders & & & $<.01$ \\
\hline Answered in-person & $72(12.2)$ & 55.6 & \\
\hline Answered by machine & $380(64.5)$ & 35.0 & \\
\hline Not completed & $116(19.7)$ & 24.1 & \\
\hline No call needed & $21(-)$ & - & \\
\hline Live phone call reminders & & & $<.01$ \\
\hline Answered in-person & $176(29.9)$ & 28.4 & \\
\hline Answered by machine & $124(21.1)$ & 12.1 & \\
\hline Not completed & $105(17.1)$ & 8.6 & \\
\hline No call needed & $184(-)$ & - & \\
\hline
\end{tabular}

FIT, fecal immunochemical test.

${ }^{*} P$ value based on likelihood ratio $\chi^{2}$ test.

${ }^{\dagger} 42$ patients in the automated arm inadvertently received live call reminders (completed in 2 to 3 weeks).

${ }^{\S}$ Patients were considered reached if the text message was sent.

- Not applicable.

than those with fewer clinic visits. Patient visits provide an opportunity for providers to recommend CRC screening, and patients with more visits may be more likely to receive such a recommendation. This finding underscores the importance of provider recommendation and/or engagement in promoting uptake of CRC screening, as observed in previous reports. ${ }^{20,22,23}$ Such recommendations may be particularly needed for Latino patients, who are reported to receive fewer in-clinic screening recommendations than their non-Latino white counterparts. ${ }^{21}$ Finally, patients who had previously completed a FIT were far more likely to return their FIT than patients who had never completed a FIT. High rates of repeat FIT testing are well-established in the literature ${ }^{12,24}$; our findings demonstrate the particular need to address barriers to CRC screening in the never-screened subgroup.

In analysis that examined FIT completion rates in patients who did and did not receive a given intervention component, we found significantly higher FIT completion rates in patients who received the automated call or live phone call but no difference in completion rates among patients who did and did not receive a text message. We previously reported that text message reminders led to lower FIT completion rates than reminder letters in a community health center population in Washington state ( $17 \%$ vs $24 \%$; OR, 0.66 ; $95 \%$ CI [0.43, 0.99]). ${ }^{14}$ Similarly, Baker and colleagues ${ }^{5}$ reported 
Table 4. Associations among Demographic and Health Care Use Characteristics and Fecal Immunochemical Test Completion, Intention-to-Treat Analysis

\begin{tabular}{|c|c|c|c|}
\hline Characteristics & FIT Completion (\%) & Unadjusted OR (95\% CI) & Multivariable Adjusted* OR (95\% CI) \\
\hline \multicolumn{4}{|l|}{ Age } \\
\hline 50 to 64 & 30.4 & Reference & Reference \\
\hline 65 to 74 & 35.7 & $1.27(0.96,1.68)$ & $0.99(0.73,1.34)$ \\
\hline \multicolumn{4}{|l|}{ Sex } \\
\hline Female & 32.9 & Reference & Reference \\
\hline Male & 29.2 & $1.19(0.97,1.46)$ & $1.04(0.83,1.29)$ \\
\hline \multicolumn{4}{|l|}{ Ethnicity } \\
\hline Non-Hispanic/Latino & 28.5 & Reference & Reference \\
\hline Hispanic/Latino & 31.9 & $1.18(0.89,1.57)$ & $0.76(0.53,1.09)$ \\
\hline \multicolumn{4}{|l|}{ Language } \\
\hline English & 25.4 & Reference & Reference \\
\hline Spanish & 35.2 & $1.59(1.29,1.97)$ & $1.39(1.07,1.82)$ \\
\hline Other & 29.4 & $1.22(0.57,2.61)$ & $1.18(0.50,2.81)$ \\
\hline \multicolumn{4}{|l|}{ Insurance } \\
\hline Medicaid/Medicare & 32.7 & Reference & Reference \\
\hline Uninsured & 29.1 & $0.85(0.66,1.08)$ & $0.76(0.58,1.00)$ \\
\hline Commercial & 29.8 & $0.88(0.67,1.15)$ & $0.84(0.63,1.13)$ \\
\hline \multicolumn{4}{|l|}{ Clinic visits in past year } \\
\hline No visits & 21.2 & Reference & Reference \\
\hline At least one visit & 33.8 & $1.90(1.44,2.51)$ & $1.67(1.22,2.30)$ \\
\hline \multicolumn{4}{|l|}{ No-shows in past year } \\
\hline No & 29.0 & Reference & Reference \\
\hline Yes & 32.4 & $1.17(0.94,1.45)$ & $0.91(0.71,1.17)$ \\
\hline \multicolumn{4}{|l|}{ Prior fecal testing } \\
\hline Never & 17.2 & Reference & Reference \\
\hline Ever & 45.1 & $3.97(3.19,4.95)$ & $3.82(3.04,4.81)$ \\
\hline \multicolumn{4}{|l|}{ Clinic } \\
\hline Clinic 1 & 32.2 & Reference & Reference \\
\hline Clinic 2 & 29.8 & $0.89(0.73,1.10)$ & $0.91(0.73,1.15)$ \\
\hline
\end{tabular}

CI, confidential interval; FIT, fecal immunochemical test; OR, odds ratio.

*Adjusted for all other variables in the model.

no difference in mailed FIT return rates among patients who did and did not receive a text message prompt or reminder, suggesting minimal influence from this approach.

Reach for prompts and reminders ranged from $51 \%$ for live calls to $81 \%$ for text messages. Our reach for text messages was slightly higher than the $51 \%$ to $78 \%$ reported in previous studies conducted in community health centers published from 2012 through 2018, 5,6,10,11,14 likely reflecting increased acceptance of use of text messaging in the 50 and older age-group. Reach of automated phone calls and live phone calls was similar to those reported in previous studies. ${ }^{5,6,25,26}$ Our participating health center has access to patient contact information from the EHR and from Medicaid Health Plans for patients enrolled in Medicaid, which may have improved the accuracy of contact information and reach of the outreach approaches we used. Nevertheless, our findings underscore the importance of maintaining updated patient contact information for optimal program delivery.

Efforts to optimize FIT completion rates might consider tailoring their approaches to address the needs of the patient subgroups found to have a relatively low response to mailed FIT outreach and reminders. Patients who have never completed a FIT or who have not had a recent clinic visit may benefit from an in-person or phone-based discussions about the importance of CRC and the need for screening. Nevertheless, such tailoring needs to be weighed against the ease of implementing the program as well as the cost. Published costs for reminders in cancer screening programs have been reported in relatively 
few previous studies, and most studies have been conducted in integrated care settings. Nevertheless, reported costs (not inflation-adjusted for differential timing) have ranged from $\$ 0.41$ to $\$ 0.50$ per live call, ${ }^{27,28} \$ 0.54$ per recipient for up to 4 automated calls, ${ }^{28,29}$ and $\$ 0.12$ per text message. ${ }^{30}$

To boost screening in the group that has never completed an FIT, our main trial will test live phone call prompts delivered before an FIT is mailed. Nevertheless, to complement centralized outreach efforts, innovations in how kits are distributed at the point-of-care may be needed. Such point-of-care efforts can complement mailed outreach approaches ${ }^{31}$; yet, more research is needed on the additive value of multilevel interventions.

\section{Strengths and Limitations}

Our study had several strengths, including its large and diverse sample size, randomized design, and the near-complete capture of FIT events and demographic characteristics in the EHR. The delivery of live reminders by bilingual (English and Spanish) clinic staff optimized the consistency of intervention delivery among those whose preferred language was English or Spanish, the predominant languages spoken by patients in our partnering health center. In addition to our study's strengths, we did have several limitations. Some aspects of our available data limited our ability to know whether a patient received the prompt or reminder: automated phone messages can be delivered to unintended recipients and we could not be certain that phones enabled text-message functions. We were limited to the data available from the vendor and have no way of knowing the magnitude of these misclassifications. Moreover, 42 patients in the automated call arm inadvertently also received a live phone call reminder. However, our sensitivity analysis showed that this error had no measurable influence on our findings. Our findings may have limited generalizability, as features at the patient level (ie, demographic characteristics and cell phone use patterns) and health center level (inclinic FIT promotion efforts) may be unique to this health center. Our study took place when the health center was using a 2-sample FIT test (before the newer 1-sample version became available), and it is likely that a 1-sample test would achieve higher return rates. Nevertheless, our primary goal was to compare the effectiveness of various mailed FIT reminders and prompts; our comparisons would likely be nondifferentially impacted by a test conversion. Finally, our analysis assessed FIT completion in a single year and we did not evaluate downstream aspects of screening and follow-up (eg, repeat FIT testing and follow-up colonoscopy).

\section{Conclusion}

Our findings showed that using live phone call reminders or a combination of automated text prompts and calls and live phone reminders resulted in a higher FIT completion rates than using automated prompts and reminders alone. Further, the overall return rate differs according to patient language preference, number of clinic visits in the past year, and prior use of FIT. Efforts to optimize FIT completion rates might consider tailoring their approaches to address the needs of these patient subgroups.

The content is solely the responsibility of the authors and does not necessarily represent the official views of the National Institute of Health. The National Institutes of Health had no role in the design and conduct of the study; collection, management, analysis, and interpretation of the data; preparation, review, or approval of the manuscript; and decision to submit the manuscript for publication.

To see this article online, please go to: bttp://jabfm.org/content/ 32/3/318.full.

\section{References}

1. Lin JS, Piper MA, Perdue LA, et al. Screening for colorectal cancer: updated evidence report and systematic review for the US Preventive Services Task Force. JAMA 2016;315:2576-94.

2. Steele CB, Rim SH, Joseph DA, King JB, Seeff LC. Colorectal cancer incidence and screening-United States, 2008 and 2010. MMWR Suppl 2013;62:53-60.

3. Meester RG, Doubeni CA, Lansdorp-Vogelaar I, et al. Colorectal cancer deaths attributable to nonuse of screening in the United States. Ann Epidemiol 2015; 25:208-13.e201.

4. Health Resources and Services Administration. 2016 National Health Center Data. Available from: https://bphc.hrsa.gov/uds/datacenter.aspx. Published 2016. Accessed November 2, 2017.

5. Baker DW, Brown T, Buchanan DR, et al. Comparative effectiveness of a multifaceted intervention to improve adherence to annual colorectal cancer screening in community health centers: a randomized clinical trial. JAMA Intern Med 2014; 174:1235-41.

6. Goldman SN, Liss DT, Brown T, et al. Comparative effectiveness of multifaceted outreach to initiate colorectal cancer screening in community health centers: a randomized controlled trial. J Gen Intern Med 2015;30:1178-84. 
7. Gupta S, Halm EA, Rockey DC, et al. Comparative effectiveness of fecal immunochemical test outreach, colonoscopy outreach, and usual care for boosting colorectal cancer screening among the underserved: a randomized clinical trial. JAMA Intern Med 2013; 173:1725-32.

8. Coronado GD, Golovaty I, Longton G, Levy L, Jimenez R. Effectiveness of a clinic-based colorectal cancer screening promotion program for underserved Hispanics. Cancer 2011;117:1745-54.

9. Coronado GD, Vollmer WM, Petrik A, et al. Strategies and opportunities to STOP colon cancer in priority populations: pragmatic pilot study design and outcomes. BMC Cancer 2014;14:55.

10. Jean-Jacques M, Kaleba EO, Gatta JL, Gracia G, Ryan ER, Choucair BN. Program to improve colorectal cancer screening in a low-income, racially diverse population: a randomized controlled trial. Ann Fam Med 2012;10:412-7.

11. Singal AG, Gupta S, Tiro JA, et al. Outreach invitations for FIT and colonoscopy improve colorectal cancer screening rates: a randomized controlled trial in a safety-net health system. Cancer 2016;122:456-63.

12. Baker DW, Brown T, Goldman SN, et al. Two-year follow-up of the effectiveness of a multifaceted intervention to improve adherence to annual colorectal cancer screening in community health centers. Cancer Causes Control 2015;26:1685-90.

13. Coronado GD, Vollmer WM, Petrik A, et al. Strategies and Opportunities to STOP Colon Cancer in Priority Populations: design of a cluster-randomized pragmatic trial. Contemp Clin Trials 2014;38: 344-9.

14. Coronado GD, Rivelli JS, Fuoco MJ, et al. Effect of reminding patients to complete fecal immunochemical testing: a comparative effectiveness study of automated and live approaches. J Gen Intern Med 2018;33:72-8.

15. Thompson JH, Davis MM, Leo MC, et al. Participatory Research to Advance Colon Cancer Prevention (PROMPT): study protocol for a pragmatic trial. Contemp Clin Trials 2018;67:11-5.

16. Norman N, Bennett C, Cowart S, et al. Boot camp translation: a method for building a community of solution. J Am Board Fam Med 2013;26:254-63.

17. Thompson JH DM, Michaels L, Rivelli JS, et al. Patient-refined Messaging for a mailed colorectal cancer screening program: application of boot camp translation in a Latino-based community health center. J Am Board Fam Med 2019;18-0026.

18. Coronado GD, Burdick T, Petrik A, Kapka T, Retecki S, Green B. Using an automated data-driven, EHR-embedded program for mailing FIT kits: lessons from the STOP CRC pilot study. J Gen Pract (Los Angel) 2014;2:1000141.
19. Petrik AF, Green BB, Vollmer WM, et al. The validation of electronic health records in accurately identifying patients eligible for colorectal cancer screening in safety net clinics. Fam Pract 2016;33: 639-43.

20. Nagelhout E, Comarell K, Samadder NJ, Wu YP. Barriers to colorectal cancer screening in a racially diverse population served by a safety-net clinic. J Community Health 2017;42:791-6.

21. Roundtable NCC. Hispanics/Latinos and colorectal cancer companion guide. Available: http://nccrt.org/ resource/hispanicslatinos-colorectal-cancer-companionguide/. Published 2016. Accessed July 27, 2018.

22. Seeff LC, Nadel MR, Klabunde CN, et al. Patterns and predictors of colorectal cancer test use in the adult U.S. population. Cancer 2004;100:2093-103.

23. Nadel MR, Berkowitz Z, Klabunde CN, Smith RA, Coughlin SS, White MC. Fecal occult blood testing beliefs and practices of U.S. primary care physicians: serious deviations from evidence-based recommendations. J Gen Intern Med 2010;25:833-9.

24. Green BB, Fuller S, Anderson ML, Mahoney C, Mendy P, Powell SL. A quality improvement initiative to increase colorectal cancer (CRC) screening: collaboration between a primary care clinic and research team. J Fam Med 2017;4:1115.

25. Hendren S, Winters P, Humiston S, et al. Randomized, controlled trial of a multimodal intervention to improve cancer screening rates in a safety-net primary care practice. J Gen Intern Med 2014;29:41-9.

26. Byrd TL, Wilson KM, Smith JL, et al. AMIGAS: a multicity, multicomponent cervical cancer prevention trial among Mexican American women. Cancer 2013;119:1365-72.

27. Schlichting JA, Mengeling MA, Makki NM, et al. Increasing colorectal cancer screening in an overdue population: participation and cost impacts of adding telephone calls to a FIT mailing program. J Community Health 2014;39:239-47.

28. Meenan RT, Anderson ML, Chubak J, et al. An economic evaluation of colorectal cancer screening in primary care practice. Am J Prev Med 2015;48: 714-21.

29. Smith DH, Feldstein AC, Perrin N, et al. Automated telephone calls to enhance colorectal cancer screening: economic analysis. Am J Manag Care 2012;18: 691-9.

30. Vidal C, Garcia M, Benito L, Mila N, Binefa G, Moreno $\mathrm{V}$. Use of text-message reminders to improve participation in a population-based breast cancer screening program. J Med Syst 2014;38:118.

31. Potter MB, Ackerson LM, Gomez V, et al. Effectiveness and reach of the FLU-FIT program in an integrated health care system: a multisite randomized trial. Am J Public Health 2013;103:1128-33. 